\title{
Beam Neutralization in the Scaled Final Focus Experiment
}

\author{
D. V. Rose, ${ }^{*}$ D. R. Welch, Mission Research Corp., Albuquerque, NM 87110, USA \\ S. A. MacLaren ${ }^{\dagger}{ }^{2}$ Lawrence Livermore National Laboratory, Livermore, CA 94550, USA
}

\section{Abstract}

A series of implicit, electrostatic, two- and threedimensional, particle-in-cell simulations are used to model heavy ion beam neutralization and focusing in the Scaled Final Focus Experiment at the Lawrence Berkeley National Laboratory. The simulations confirm the charge neutralizing effect of electrons introduced into the beam path as the beam is focused and exiting the accelerator.

\section{INTRODUCTION}

The Final Focus Scaled Experiment [1] (FFSE) at Lawrence Berkeley National Laboratory is designed to study vacuum ballistic focusing of a heavy ion beam. The dimensional parameters are one-tenth scale values of the HIBALL-II heavy ion driver design [2] and the beam dynamics parameters are then scaled to preserve the emittance and perveance [1]. In one set of experiments, the beam current was raised to approximately four times the scaled value in order to examine the role of electron-neutralization of the beam space-charge.

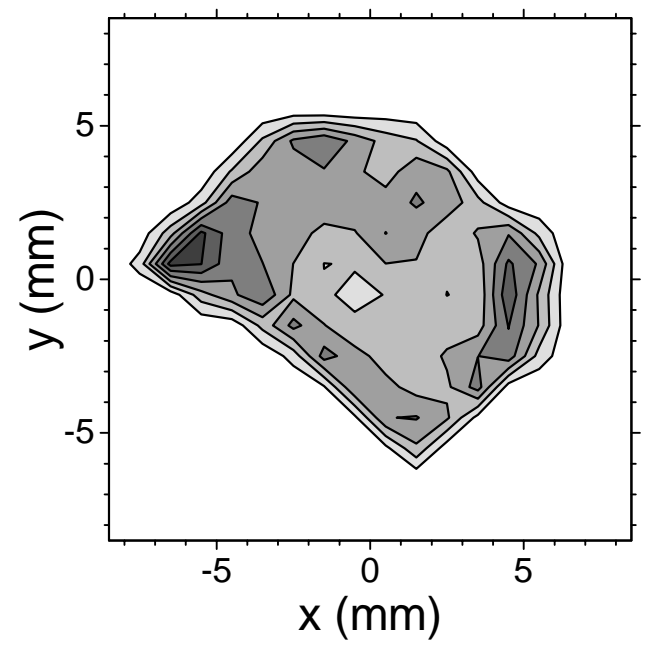

Figure 1: Plot of the measured beam current density exiting the accelerator (time-integrated) near the middle of the beam pulse, prior to focusing. Contour levels are linear with darker shades denoting higher current density.

In the experiment, the $400 \mu \mathrm{A}, 160 \mathrm{keV} \mathrm{Cs}^{+1}$ ion beam is brought to a focus $80 \mathrm{~cm}$ from the exit of the last quadrupole. The beam spatial profile is measured with a thin slit diagnostic and the beam pulse duration is $10 \mu \mathrm{s}$. The accelerator generates a highly reproducible beam with

\footnotetext{
*drose@mrcabq.com

$\dagger$ maclaren2@1lnl.gov
}

a non-circular cross-section, as shown in Fig. 1. This data is taken from near the center of the beam pulse and shows that the current-density is non-uniform across the beam.

The source of electrons used in neutralized beam runs is a glowing tungsten filament placed across the beam path. The effective filament temperature of $\sim 2100 \mathrm{~K}$ provides a dense, ready source of cool electrons $(\sim 0.2 \mathrm{eV})$ in the beam path. The $75 \mu \mathrm{m}$ thick filament intercepts less than $1 \%$ of the total beam current [1].

The horizontal and vertical beam profiles from the neutralized and unneutralized beams were obtained from a slit diagnostic at the focal position. The slit width is $0.05 \mathrm{~mm}$, and the step size between measurements is $0.25 \mathrm{~mm}$ for the unneutralized beam and $0.1 \mathrm{~mm}$ for the neutralized beam.

\section{SIMULATION MODEL}

Two-dimensional, axisymmetric, electrostatic particlein-cell (PIC) simulations were carried out first. All simulations described here use the LSP code [3, 4]. The implicit field and particle algorithms were used for these long time scale $(>2 \mu \mathrm{s})$ simulations. The simulation region is 120 $\mathrm{cm}$ long $(L)$ and $3 \mathrm{~cm}$ in radius $(R)$, enclosed by conducting walls. The injected beam is assumed to have the axisymmetric (tophat) current density profile shown in Fig. 2. This profile was obtained by averaging eight radial profiles originating from the beam center-of-mass. (The beam centerof-mass was determined from Fig. 1 to be $x \simeq-0.21 \mathrm{~mm}$ and $y \simeq+0.3 \mathrm{~mm}$.) The beam ions have a $67-\mathrm{cm}$ focal length (distance of the experimental diagnostic), and then an additional angle is randomly added to each ion to model the emittance [1]. The source of neutralizing electrons was modeled by space-charge limited emission from the beam entrance plane $(z=0)$. The field-emission threshold was set to $0.01 \mathrm{~V} / \mathrm{cm}$, much less than the beam space-charge fields, enabling a nearly continuous supply of electrons to be pulled from the beam injection boundary.

The beam is injected from the left boundary for $2.4 \mu \mathrm{s}$. The injected beam ions are $160 \mathrm{keV} \mathrm{Cs}^{+1}(\beta=0.0016)$, with a current that rises linearly from zero to $400 \mu \mathrm{A}$ in $200 \mathrm{~ns}$ and then remains constant. The beam density and electric field profiles were analyzed at $z=67 \mathrm{~cm}$.

Previous work has shown that ion beams neutralized by co-moving electrons are unstable [5]. This Pierce-type instability has a current threshold (in the non-relativistic limit) of $I_{t} \simeq 3\left[1+(1.31 R / L)^{2}\right] I_{l}$, where the nonrelativistic electron space-charge-limiting current [6] is $I_{l}(A) \simeq$ $66 \times 10^{-6} V^{3 / 2}$, and $V=m_{e} \beta^{2} c^{2} / 2 e$. For the FFSE parameters, the beam current exceeds the threshold $I_{t} \simeq$ $100 \mu \mathrm{A}$. Neutralization degraded significantly in the simulations after the beam reached the downstream conducting 


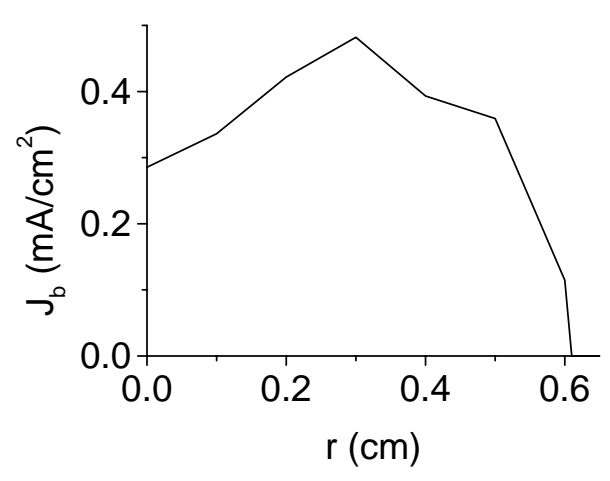

Figure 2: Radial current density profile used in the 2-D simulations.

boundary $(t>2.5 \mu \mathrm{s})$.

\section{SIMULATION RESULTS}

Table 1 lists the 2-D simulations that were run to examine the neutralization of the $400 \mu \mathrm{A}$ FFSE beam. The first simulation modeled complete neutralization of the beam by turning off self-forces in the simulation; i.e., the injected beam ballistically drifted through the simulation volume. This "orbit" calculation gives a lower limit on the beam spot size at the target plane (no net pinching forces are expected for this beam perveance). The second simulation assumed no electron sources and the beam expanded as it propagated due the unneutralized beam space charge. The third simulation used the space-charge-limited emission model discussed in Sec. 2 to provide neutralizing electrons. Table 1 also lists the RMS radius of the beam at $z=67 \mathrm{~cm}$ and the radial and axial electric field at $z=67 \mathrm{~cm}, r=r_{R M S}$ and $t=2.4 \mu \mathrm{s}$.

Table 1: 2-D LSP simulation results. The electric field values and beam RMS radii are taken at $z=67 \mathrm{~cm}$.

\begin{tabular}{|c|c|c|c|c|}
\hline Run & Neutralization & $\begin{array}{c}r_{r m s} \\
(\mathrm{~mm})\end{array}$ & $\begin{array}{c}E_{r} \\
(\mathrm{~V} / \mathrm{cm})\end{array}$ & $\begin{array}{c}E_{z} \\
(\mathrm{~V} / \mathrm{cm})\end{array}$ \\
\hline sm10 & Complete & 0.84 & - & - \\
sm11 & None & 2.72 & 31 & 0.5 \\
sm12 & Partial & 0.95 & 57 & -3.0 \\
\hline
\end{tabular}

The integrated beam charge profiles at $z=67 \mathrm{~cm}$ from the three simulations are shown in Fig. 3. For the complete and partially neutralized simulations, the entire beam is within $2 \mathrm{~mm}$, while the unneutralized beam has a much broader profile. Thus, the wall emitted electrons provide a very high degree of charge neutralization.

For direct comparison of the simulation results with the experimental measurements, the radial charge density profiles from the simulations were converted to slit measurements similar to the diagnostic used in the experiment. These comparisons are shown in Fig. 4. The individual curves are normalized by their areas. The solid lines denote

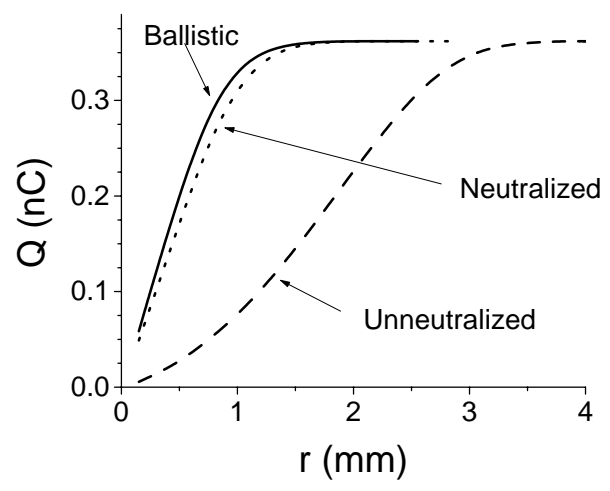

Figure 3: Time-integrated beam charge as a function of radius from the 2-D simulations at $z=67 \mathrm{~cm}$ and $t=2.4$ $\mu \mathrm{s}$.

the simulation results and dashed lines denote the experimental measurements. Comparisons in both the vertical and horizontal measurement planes were made, but only the vertical plane experimental results are shown here. The experimental curves are shifted by less than $1 \mathrm{~mm}$ to obtain the best comparison with the simulation results. For both the unneutralized and unneutralized beams, good agreement is found over most of the beam widths.

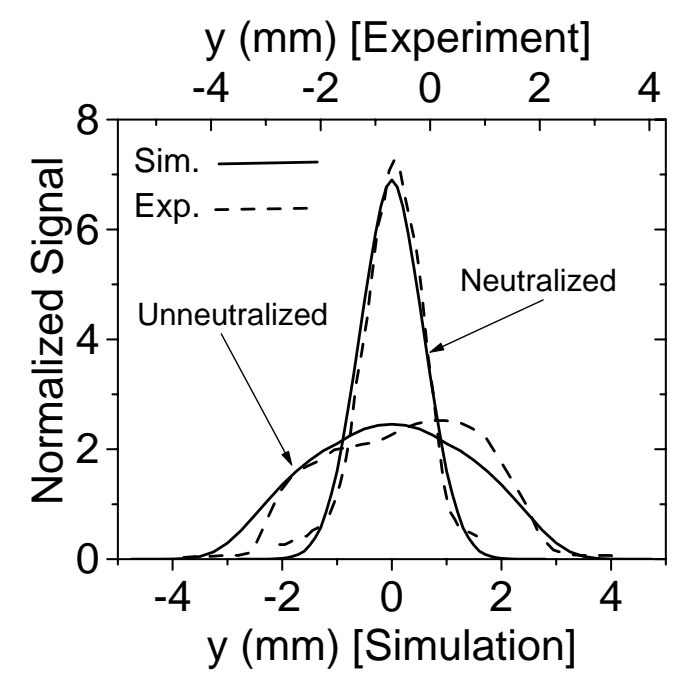

Figure 4: Comparison of the simulated (solid line) and experimental (dashed line) slit diagnostic measurements, vertical plane.

In both vertical and horizontal planes, the neutralized beams are significantly smaller than the unneutralized beams, demonstrating the almost complete elimination of the space-charge spreading of the beam when the hot filament was introduced into the beam path. For the neutralized case, the experimental result does appear to be slightly narrower than the simulated result, possibly indicating better overall charge neutralization than was obtained in the simulation. Differences are more likely due to the asymmetric phase-space distribution of the injected beam whose 
spatial distribution is shown in Fig. 1.

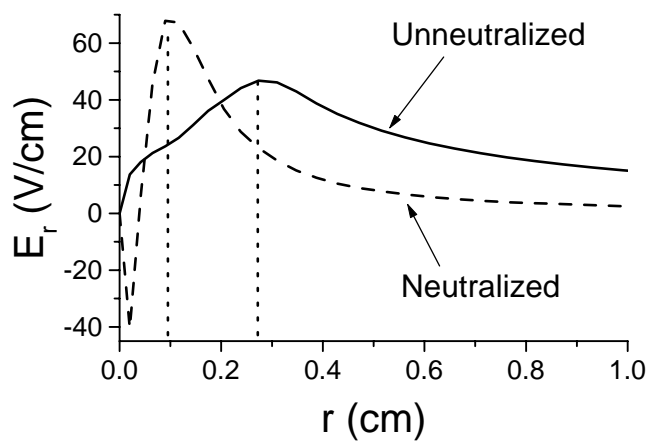

Figure 5: Profiles of the radial electric fields at $z=$ $67 \mathrm{~cm}$ from the unneutralized (solid lines) and neutralized (dashed) simulations. The dotted lines indicate the beam RMS radius.

In Fig. 5, the radial electric fields from the simulations are shown at $z=67 \mathrm{~cm}$ and $t=2.4 \mu \mathrm{s}$. The beam RMS values given in Table 1 occur at slightly smaller radii than the peak radial electric field values in both simulations. These field profiles indicate that the neutralization fraction at the focus for the neutralized beam is approximately $85 \%$.

Electrons co-moving with the beam are expected to heat as the beam compresses radially (see, for example Ref. [7]). The electron speed distributions for electrons in the vicinity of the beam head are shown in Fig. 6 at 0.4, 0.8, 1.2, and $1.6 \mu \mathrm{s}$. This data is taken from the neutralized simulation, and these times correspond to beam head positions of $19,38,58$, and $77 \mathrm{~cm}$. These distributions indicate that the electrons gradually heat as the beam head approaches the focal position at $z=67 \mathrm{~cm}$. The electrons are heated significantly as the beam reaches the minimum radius.

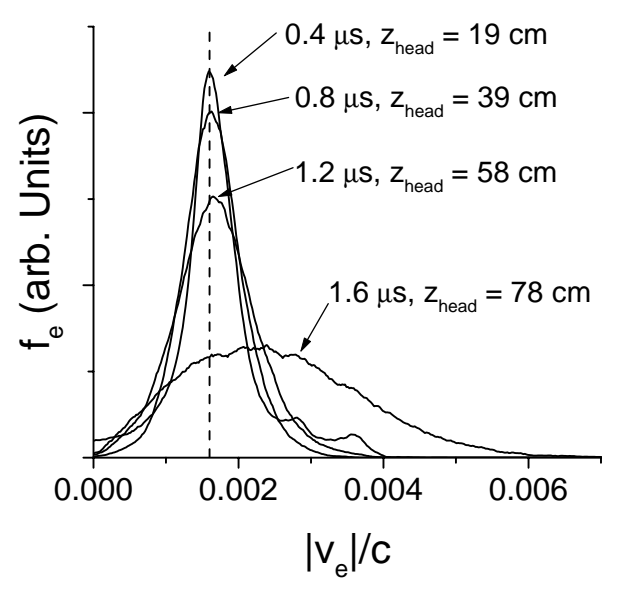

Figure 6: Electron speed distribution from the neutralized simulation (sm12) at several times. The vertical dashed line indicates the ion beam speed.

Examination of the total electron population in the simulation as a function of time indicates that the bulk of the electron distribution moves at roughly the beam speed.
However, a hot tail population is observed to grow after the beam head has passed through the focal plane. The electron radial velocity distribution remains essentially symmetric throughout, but the positive axial electron distribution heats in time (before the beam reaches the downstream wall). The nature of this growing "tail" population may be due to the two-streaming interaction of the beam and electron populations [8]. More detailed numerical simulations are needed to quantify this.

3-D simulations using the 2-D beam spatial profile from Fig. 1 were also carried out to examine measured beam asymmetries at the focal plane. These simulations (both ballistic and unneutralized) did not produce any significant differences in the beam profiles at the focal position when compared with the 2-D axisymmetric simulations described above. This suggests that the details of the beam phase-space are responsible for the asymmetric features in the measured beam profiles.

\section{DISCUSSION}

The PIC code LSP has been used to examine beam neutralization in the FFSE. Simple assumptions about the injected beam distribution and electron source demonstrate the effect of charge neutralization on the beam spatial distribution at the focal plane. The results presented here confirm the conclusion of the FFSE in that electron neutralization is responsible for the observed enhancement in beam focusing. An axial supply of electrons can neutralize down to a minimum potential of $m_{e} \beta^{2} c^{2} / 2 e$ [9]. Here, this minimum potential is $\simeq 0.7 \mathrm{~V}$, while the unneutralized beam potential is approximately $7.5 \mathrm{~V}$. This indicates a neutralization fraction of approximately $90 \%$, consistent with the results obtained in the simulations.

The authors acknowledge helpful discussions with Dr. A. Friedman and Dr. C. Olson. Work funded by the US DOE through Lawrence Berkeley National Laboratory and Princeton Plasma Physics Laboratory.

\section{REFERENCES}

[1] S. MacLaren, et al., Nucl. Inst. Meth. Phys. Res. A 464, 126 (2001).

[2] B. Badger, et al., Report No. UWFDM-625, Univ. Wisconsin (1984); and Report No. Kfk 3840, Kernforschungszentrum Karlsruhe (1985), p. 57.

[3] T. P. Hughes, et al., Phys. Rev. ST-AB 2, 110401 (1999).

[4] D. R. Welch, et al., Nucl. Inst. Meth. Phys. Res. A, 464, 134 (2001).

[5] J. W. Poukey, et al., J. Appl. Phys. 52, 3016 (1981).

[6] C. L. Olson and J. W. Poukey, Phys. Rev. A 9, 2631 (1974).

[7] D. V. Rose, et al., Mission Res. Corp. Report MRC/ABQ-R2025 (2001).

[8] R. N. Sudan, Phys. Rev. Lett. 37, 1613 (1976).

[9] C. L. Olson, et al., Fus. Eng. Design 32-33, 485 (1996). 